\title{
A COMPUTER MODEL OF GLOBAL THERMOSPHERIC WINDS AND TEMPERATURES
}

\author{
T. L. Killeen, ${ }^{*}$ R. G. Roble ${ }^{* *}$ and N. W. Spencer ${ }^{* * *}$ \\ * Space Physics Research Laboratory, The University of Michigan, Ann Arbor, \\ MI 48109, U.S.A. \\ ${ }^{* *}$ High Altitude Observatory, National Center for Atmospheric Research, $\dagger$ \\ Boulder, CO 80307, U.S.A. \\ ***Goddard Space Flight Center, Greenbelt, MD 20771, U.S.A. \\ tThe National Center for Atmospheric Research is sponsored by the National \\ Science Foundation
}

\section{ABSTRACT}

A computer model of the global, time-dependent, thermospheric horizontal vector neutral wind and neutral temperature fields has been constructed based on output from the NCAR thermospheric general circulation model (NCAR-TGCM). The wind field is represented by a vector spherical harmonic (VSH) expansion in the horizontal, a fourier expansion in Universal Time, and a polynomial expansion in altitude. The global temperature field representation differs in that a scalar spherical harmonic expansion is used in the horizontal and a Bates model temperature profile is used in altitude. A set of suitably-truncated spectral coefficients contains the wind and temperature description for a diurnally-reproducible run of the NCAR-TGCM. The VSH model is coded in a FORTRAN subroutine that returns vector wind and temperature values for a given UT, geographic location, and altitude. The model has applicability for studies of thermospheric and/or ionospheric physics where reasonable timedependent neutral wind and temperature values are of interest. The routine is novel since portable computer models of thermospheric wind fields have not previously been available to researchers. The current version of the model is valid for solar maximum, December solstice only, although the model can be extended to any season and specific set of geophysical conditions for which TGCM results are available. Results from the VSH computer model are presented to compare with global-scale wind measurements from the Dynamics Explorer (DE-2) satellite. The agreement between the computer model results and data from individual orbits of DE-2 is good, indicating that the model provides reasonable wind values, having the appropriate characteristic latitudinal, diurnal, and Universal-Time-dependent signatures observed from the satellite at upper thermospheric altitudes. The VSH thermospheric temperature values are in general agreement with MSIS-83 temperatures but illustrate smaller-scale horizontal temperature structures than are resolved by MSIS-83, owing to the larger number of spectral harmonics retained.

\section{INTRODUCTION}

Significant progress has been made over the last several years in the modeling and empirical description of the global thermospheric neutral wind and temperature system. The Dynamics Explorer (DE 2) spacecraft, in particular, was instrumented to measure the thermospheric vector wind and temperature along the track of the polar-orbiting spacecraft $/ 1-3 /$. Published results from this mission have served to characterize the global-scale thermospheric wind field for the solar maximum conditions pertaining to the 1981-1983 period /3-9/. In addition to the new information provided by the DE 2 spacecraft and other experimental techniques, the theoretical understanding of thermospheric motions has progressed rapidly. Various theoretical models exist that can simulate the dynamical response of the upper atmosphere for a variety of geophysical conditions. In particular, two numerical general circulation models, the NCAR-TGCM $/ 10,11$ / and the UCL-TGCM $/ 12$ / have had a large measure of success in calculating wind and temperature fields similar to those observed from DE $2 / 6,9,13-17 /$. The spectral model of Mayr et al. $/ 18 /$ has also provided significant additional insight into global-scale thermospheric dynamics.

In spite of these recent theoretical modeling efforts, no simple "user-friendly" computer model of the global thermospheric wind field has been previously constructed to enable neutral winds to be conveniently used in other theoretical studies or in straightforward comparisons with new data sets. The underlying reasons for this situation involve the sophistication and complexity of the TGCMs and the large physical size of the data arrays necessary to contain the numerically-simulated wind fields. Moreover, the fragmentary nature of the global-scale wind measurements collated to date from all experimental sources has postponed the construction of a purely empirical model. For thermospheric temperatures, the situation is better in that semi-empirical models, such as the MSIS-83 model of Hedin /19/, have, for many years, provided researchers with reliable values for thermospheric temperatures, incorporating explicit dependences on geomagnetic activity, solar activity, and season.

The purpose of this report is to describe a new computer model of the global, horizontal, thermospheric vector wind and neutral temperature structure from 130 to $600 \mathrm{~km}$ altitude. We call it the VSH model, since it contains a description of the wind field using vector spherical harmonics. The new model is based on a spectral expansion of the gridded output wind and temperature fields provided by specific runs of the NCAR-TGCM. As such, the physical description provided by the VSH model is determined by the physical, chemical and dynamical processes contained within the NCAR-TGCM structure. The VSH model formulation does not, as yet, include an explicit dependence on season, solar cycle or geomagnetic activity level. It does, however, allow for such effects to be catered for through generation of separate sets of VSH model coefficients, each of which describes specified geophysical conditions and shares a common retrieval subroutine.

The VSH model is coded in a FORTRAN subroutine that returns vector wind and temperature values for a given UT, geographic location, and altitude. It, therefore, has applicability for many studies of thermospheric and/or ionospheric physics where reasonable, UT-dependent neutral wind profiles or single-point values are of interest. We note that portable computer models of thermospheric wind fields have not previously been available to researchers. The VSH model temperature values are of interest for two reasons. Firstly, they enable the temperature predictions of the NCAR-TGCM to be made generally available for comparison with measurements and semi-empirical models. Secondly, since the number of spectral coefficients retained in the VSH representation is large compared with MSIS-83 and other semi-empirical models, the VSH temperature fields contain localized temperature structures, such as cusp temperature enhancements, that are commonly filtered out of the semi-empirical models. This last feature, of course, comes at the expense of computer time (see below). 
The current version of the VSH model is valid for solar maximum, December solstice conditions only, although the model can be extended to any season and any set of geophysical conditions for which TGCM results are available. The formulation of the VSH model has been designed to allow for experimental data to be included in the fitting procedure, enabling the future development of a semi-empirical model of thermospheric winds through the suitable merging of experimental measurements with the TGCM gridded predictions.

In section 2, we describe the formulation of the new computer model. In section 3, we present results from the model or a solar maximum, December solstice case corresponding to moderately active geomagnetic conditions, and compare these with 1) global-scale wind measurements from the DE 2 spacecraft and 2) temperature profiles from the MSIS-83 semi-empirical model. In section 4 we summarize our results and discuss the utility of the VSH model and future planned developments.

\section{VSH MODEL FORMULATION}

The basis for the VSH model is a spectral expansion of output from the NCAR-TGCM. The expansion is performed as part of the TGCM diagnostic package described by Killeen and Roble /20/. We briefly discuss the TGCM and the specific output used in the present work before describing the formulation of the VSH model.

The NCAR-TGCM has been discussed in detail in a series of papers /10,11,13,14/and here we review only the basic features. The TGCM solves the hydrodynamic, thermodynamic and continuity equations appropriate to the Earth's thermosphere for a given set of geophysical, time-dependent input conditions and stores the calculated wind, temperature and composition (mass mixing ratio) output fields at selected Universal Times (UTs) during the model run. The model has a $5^{\circ}$ latitude-by-longitude grid with 24 constant-pressure surfaces in the vertical, extending from approximately 97 to $500 \mathrm{~km}$ in altitude. The version of the TGCM used in this work incorporates the coupling of dynamics and composition $/ 2 \mathrm{l} /$ and calculates the solar heating distribution and $\mathrm{O}_{2}$ photodissociation rates using the procedure described by Dickinson et al. /10,21/. The Hinteregger/22/solar EUV flux values and the Torr et al. 123/ solar UV flux values are used to provide the direct solar input corresponding to the geophysical conditions appropriate to the particular model run. The ion-convection model of Heelis et al. $/ 24 /$ is used for the specification of high-latitude ion drifts. For the examples discussed here, the Heelis et al. model input parameters were chosen to yield a Volland-type symmetric ion-convection geometry. The Chui $125 /$ model of ionospheric densities is supplemented by auroral particles according to the prescription of Roble et al. $/ 26 /$ to provide the ion-drag tensor values necessary for the calculation of both the ion-drag momentum source and the Joule heat source to the thermosphere. The auroral oval used is similar to the statistical patterns described by Spiro et al . 27/ and Whalen 28/. The TGCM run used to construct the present VSH computer model has been employed previously in several theoretical studies and for comparisons with DE-2 data $/ 6,8,28 /$. It is, therefore, well documented and we refer readers to these papers for more detailed information.

The TGCM diagnostic processor $/ 20$ / is exercised following the basic TGCM model run. It reads in the history file produced by the TGCM as well as other relevant input parameters and then proceeds to calculate diagnostic information at selected model grid points and UTs. The spectral analysis capability of the diagnostic package, to be described in more detail in a forthcoming paper, is used to provide the coefficients for the VSH model (see below). The history file contains records of the global wind, temperature and composition fields calculated by the TGCM at each hour of UT for the 24 hour simulation. Geophysical conditions corresponding to December solstice, solar maximum, and moderately active geomagnetic conditions $(\mathrm{Kp} \sim 3)$ were used for the specific TGCM run that has provided the coefficients for the first version of the VSH model, presented here. The TGCM was run until diurnal-reproducibility was attained, i.e., "steady-state," diurnally-modulated forcings were used.

For the purpose of the horizontal wind expansion, the gridded vector wind predictions at each of 24 UTs and at each of three constant-pressure levels $(z=-4$, corresponding to $\sim 130 \mathrm{~km}$ altitude; $z=-1$, corresponding to $\sim 250 \mathrm{~km} ; \mathrm{z}=1$, corresponding to $\sim 400 \mathrm{~km}$ ) are expanded using vector spherical harmonic functions which are the appropriate eigen-functions for vector fields on the sphere. This expansion has the following form:

$$
V=\Sigma\left(a_{m, n} P_{m, n}+b_{m, n} B_{m, n}+c_{m, n} C_{m, n}\right)
$$

where $V$ is the vector wind field and $a_{m, n}, b_{m, n}, c_{m, n}$ are the complex vector spherical harmonic coefficients; $m$ is the zonal harmonic (order) and $n$ is the degree. Here,

$$
\begin{aligned}
P_{m, n} & =\left[\begin{array}{c}
P_{n}^{m} \\
O \\
O
\end{array}\right] e^{i m \phi} ; \\
B_{m, n} & =\left[\begin{array}{c}
O \\
A_{n}^{m} \\
i B_{n}^{m}
\end{array}\right] \frac{e^{i m \phi}}{\sqrt{n(\overline{n+1})}} ; \\
C_{m, n} & =\left[\begin{array}{c}
0 \\
i B_{n}^{m} \\
-A_{n}^{m}
\end{array}\right] \frac{e^{i m \phi}}{\sqrt{n(\overline{n+}-1)}} .
\end{aligned}
$$

where $\emptyset$ is east longitude, and $A_{n}^{m}$ and $B_{n}^{m}$ are functions of $\theta$ (colatitude) only:

$$
\begin{aligned}
& A_{n}^{m}=\frac{d P_{n}^{m}}{d \theta} \\
& B_{n}^{m}=\frac{m}{\sin \theta} P_{n}^{m}
\end{aligned}
$$

$P_{n}^{m}$ are the associated Legendre functions given by

$$
P_{n}^{m}(\theta)=\frac{1}{2^{n} n !}(\sin \theta)^{m} \frac{d^{(m+n)}}{d x^{m+n}}\left(x^{2}-1\right)^{n} ; \quad x=\cos \theta .
$$


The complex coefficients are obtained using a least-squares fit of the TGCM output winds to the above formula. Conversely, once the coefficients are available, the global wind field can be readily reconstituted in whole or in part, using equation 1 . Since the resolution of the TGCM grid is 5 degrees, only those coefficients for which $0<n<37$ and $0<m<n$ are of significance. We note that equation 1 is an expression for the full vector wind, including the radial (vertical) component involving $\mathbf{P}_{\mathrm{m}, \mathrm{f}}$. While the two horizontal wind components are coupled via the functions $A_{n}^{m}$ and $B_{n}^{m}$, the vertical component is uncoupled and, therefore, can be simply expressed in terms of a (scalar) spherical harmonic expansion involving the associated Legendre functions. Since the vertical winds calculated by the TGCM are small in magnitude and, for some applications, not of particular interest, we (optionally) substitute for the vertical wind a sealar field such as temperature or mass mixing ratio and use the coefficients $a_{m}$ to describe the selected scalar field conveniently within our three-dimensional vector spherical harmonic representation. For the present VSH model, we choose to fit for neutral temperature to provide a direct comparison with the MSIS-83 model. The complete array of coefficients for the fit represents a large set of numbers, commensurate in size to the history file record of winds and temperatures itself. Thus, the desired reduction in the size of the set of numbers describing the output TGCM fields can only be attained by truncating the coefficients. Such truncation, of course, tends to destroy progressively the fidelity with which the wind fields can be reconstituted, and must be carried out carefully to ensure that important morphological features in the thermospheric wind pattern are not lost unwittingly.

Figure 1 presents contours of the log (base 10) amplitude for the VSH coefficients (real and imaginary) calculated for the spectral expansion of horizontal winds on the $\mathrm{z}=1$ and $\mathrm{z}=-4$ constant pressure surfaces at 1 200UT. As mentioned above, the specific TGCM run was chosen since it corresponds to the geophysical conditions for which much of the DE 2 data applies. In the figure, the bottom right triangular section is unfilled since only coefficients with $0<\mathrm{m}<\mathrm{n}$ are non-zero. As can be seen, the amplitude and, therefore, the power in the expansion is a maximum at the lower wavenumbers. The amplitudes tend to diminish in magnitude with increasing wavenumber, as would be expected, though the amplitude drops much more rapidly with increasing $\mathrm{m}$ than with increasing $\mathrm{n}$. The amplitudes for the $\mathrm{z}=-4$ surface are smaller in magnitude than for the $\mathrm{z}=1$ surface since the wind speeds are generally much reduced at the lower altitudes (e.g., Roble et al., /11/). The shape of the contours shown in figure 1 provides the key to the design of a suitable truncation scheme to reduce the mass of numbers required for the spectral representation of the thermospheric wind field.
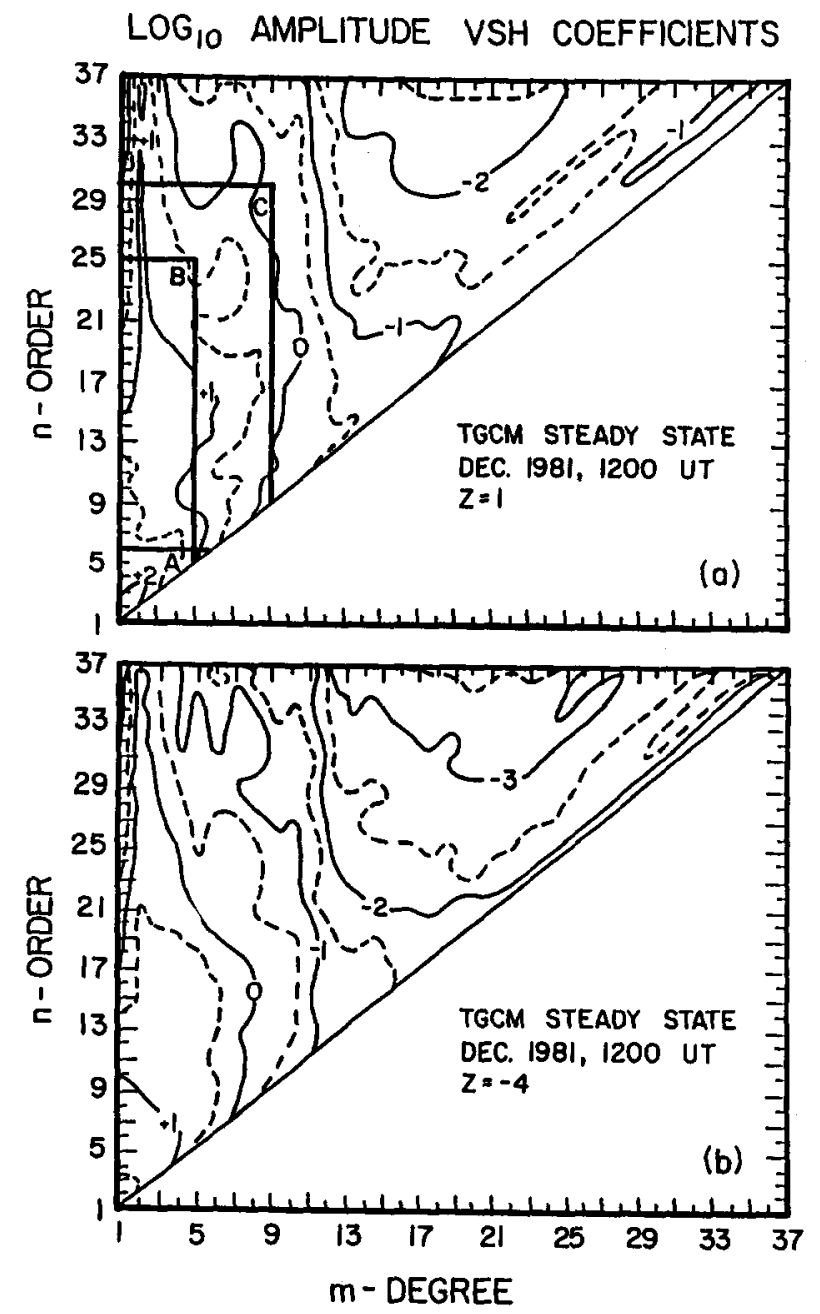

Fig. 1. Contours of the logarthmic amplitude (base 10) for the vector spherical harmonic coefficients calculated by fitting the global thermospheric wind field calculated by the NCAR-TGCM are plotted as a function of degree and order for the geophysical conditions discussed in the text and for 1200UT. Figure la is for the $\mathrm{z}=1$ constant-pressure surface and Figure $1 \mathrm{~b}$ for the $\mathrm{z}=$ -4 constant-pressure surface. Shown in Figure la are three regions illustrating various levels of truncation; level B is used for the VSH model. 
In setting the level of truncation for the VSH model we used the stringent criterion that the high-latitude reversals and vortices in the neutral wind pattern associated with jonospheric convection should be retained. It was found that a rectangular truncation scheme in $n-m$ space gave excellent results in terms of the fidelity of the reconstituted high-latitude wind field for the minimum number of coefficients. Three levels of truncation are indicated in Figure la. Level A corresponds to the number of harmonics commonly retained for semi-empirical models (e.g., MSIS-83) employing scalar spherical harmonics. Level C (i.e., truncating the coefficients at $\mathrm{n}=30, \mathrm{~m}=9$ ) was found to provide almost perfect fidelity for the neutral wind reconstitution. For the VSH model, we employ truncation at level $B(n=25, m=5)$, which represents a good compromise between minimizing the size of the retained coefficient array (and therefore the computational time for wind syntheses) and keeping the required high-latitude wind structure. Note that the empirically-optimized truncation scheme favored higher values of $\mathrm{n}$ over $\mathrm{m}$. This is due to the relatively large amplitudes of the fitted coefficients for large $n$, small $\mathrm{m}$; these particular coefficients contain the information necessary to describe the relatively small-scale high-latitude neutral wind structure.

The VSH expansion, discussed above, is only for a single constant-pressure level and for a given UT. To extend the expansion to include the temporal evolution of the wind field over the 24 hour diurnal TGCM simulation, we repeat the vector spherical harmonic expansion for all 24 hourly history file records and then perform a conventional complex fourier time series to fit to the individual VSH coefficients. We have found that the fourier coefficients so obtained may be truncated such that only seven (including odd and even fourier coefficients) need to be stored to describe the diurnal variation of the "steady-state" neutral wind field reasonably well, incorporating the well-known "UT effects" associated with the diurnal revolution of the geomagnetic pole about the geographic pole. Clearly, for a situation where the thermosphere is disturbed, such as during a geomagnetic storm, additional fourier coefficients would be required to describe the more complex time-dependences. The fourier series truncation used here, however, is adequate for the diurnally-reproducible case. The final part of the expansion deals with the altitudinal structure of the winds and temperatures. Since the variation in altitude of the neutral thermospheric horizontal wind predicted by the NCAR-TGCM is relatively smooth (see, for example, figure 12 of Sica et al, $/ 29 /$, we complete the wind expansion by fitting the fourier time series coefficients to a simple, second order polynomial in altitude. The conversion from constant-pressure levels to altitudes is made using the calculated global averages for the heights of the specific constant-pressure levels carried forward to the VSH model. This conversion introduces small inconsistencies between the TGCM and VSH wind predictions that are not considered to be of importance for the stated purposes of the present work. In the case of the wind altitudinal variation, an additional constraint is placed on the VSH altitude profile to force the individual wind component values to reach constant (i.e., exospheric) values above $\sim 400 \mathrm{~km}$. This constraint is justified, on theoretical grounds, by the high kinematic viscosity of the atmosphere at these heights (which tends to reduce vertical wind shears) and, on experimental grounds, by the DE 2 data which show little altitudinal structure above $\sim 400 \mathrm{~km}$.

For temperatures, we replace the polynomial altitude expansion with a two-parameter fit to a Bates model profile $/ 30,31 /$ having the appropriate monotonic form, with the asymptotic (exospheric) temperature and scale-height parameter given by the fit.

The three expansions, namely, the VSH expansion in the horizontal (including the scalar spherical harmonic expansion for temperature), the fourier series in time, and the polynomial (or Bates profile) expansion in altitude, complete the full description of the TGCM wind and temperature simulations. The full set of numbers containing this description at the maximum accuracy would comprise an array of dimensions $37 \times 18 \times 6 \times 25 \times 24$, corresponding to, respectively, the order $(n)$, the degree $(m)$, the components of the complex coefficients $(a, b$, and $c)$, the fourier time series, and the altitude polynomial. With the truncation levels discussed above, we reduce the coefficient array size to the dimensions $25 \times 5 \times 6 \times 7 \times 3$. Thus a total of 15750 coefficients are stored for the VSH model. We consider this to be close to the minimum number required to describe the full diurnal, spatial and altitudinal variation of the thermospheric vector neutral wind and temperature fields using spherical harmonics while retaining the important high-latitude wind structures (vortices and reversals) observed from DE 2. It might be possible in the future to further reduce the size of the required coefficient array by choosing a different set of orthonormal functions for the expansion, optimized to provide high spatial frequencies at high latitudes (A.D. Richmond, 1985, private communication).

The VSH model consists of the stored set of coefficients (occupying approximately 300 blocks of disk space on a VAX or PDP-type computer), plus a subroutine designed to reconstitute the vector wind and temperature values at any given geographic location, altitude and time. The subroutine performs the inverse set of transformations to those that provided the coefficients, recovering, successively, the temporal, spatial, and altitudinal information to return geophysical winds and temperatures. The computer time necessary for this "synthesis" of model predictions is significantly longer than for similar calculations using MSIS-83, due to the much larger number of calculations necessary. We have found that full global 5 degree $\times 5$ degree wind and temperature fields can be calculated using the VSH model within a few minutes CPU time on a VAX 750 machine. Much shorter computer times, however, are required to calculate, for example, the diurnal variation of the neutral winds over a given ground-based observatory. For most applications, computer time can be reduced considerably by using the most efficient nesting of DO loop calls to the subroutine.

In the next section we present various results from the VSH model to illustrate its current capabilities. The VAX FORTRAN subroutine and documentation necessary for its use can be obtained from one of the authors (TLK).

\section{EXAMPLES OF RESULTS FROM THE VSH MODEL}

As mentioned earlier, the present version of the VSH model was designed to correspond with much of the DE 2 data base. In figures $2 \mathrm{a}(\mathrm{b})$, we show north (south) polar projections illustrating VSH model vector winds (in cyan) calculated for an altitude of $400 \mathrm{~km}$ at the appropriate Universal Time corresponding to orbit $1819(7438)$ of DE 2. The VSH winds are overlaid with the measured vector wind measurements from DE 2 (in yellow) made at altitudes of $\sim 400 \mathrm{~km}$ by the Fabry-Perot interferometer, FPI, $/ 1 /$ and the Wind and Temperature Spectrometer, WATS, $/ 2 /$. The DE 2 vector winds are generated from the measurements of these two instruments using the data-merging technique of Killeen et al. /3/. The DE 2 data shown were selected on the basis of the moderate-to-active levels of geomagnetic activity existing at the time of the orbital passes (see $\mathrm{Kp}$ values indicated). In both northern and southern cases, excellent qualitative agreement between the VSH model winds and the DE 2 measurements is evident, with the UT-dependent locations of the various high-latitude morphological wind features well described by the model. While exact agreement between individual DE 2 measurements and VSH model values is not obtained, nor expected, the VSH winds clearly provide reasonable results that are directly comparable with the observations, both in magnitude and direction. They are also useful in providing a hemispheric context for the interpretation of the DE 2 measurements.

The results shown in Figure 2 indicate that the various high-latitude features built into the NCAR-TGCM, such as the differing offsets between geographic and geomagnetic poles in the two hemispheres and the dominating influence on the neutral wind pattern of ion drag associated with the twin vortex ionospheric convection, are reflected in the VSH computer model. It is, of course, these high-latitude features that determine the VSH coefficient truncation criteria discussed above. 

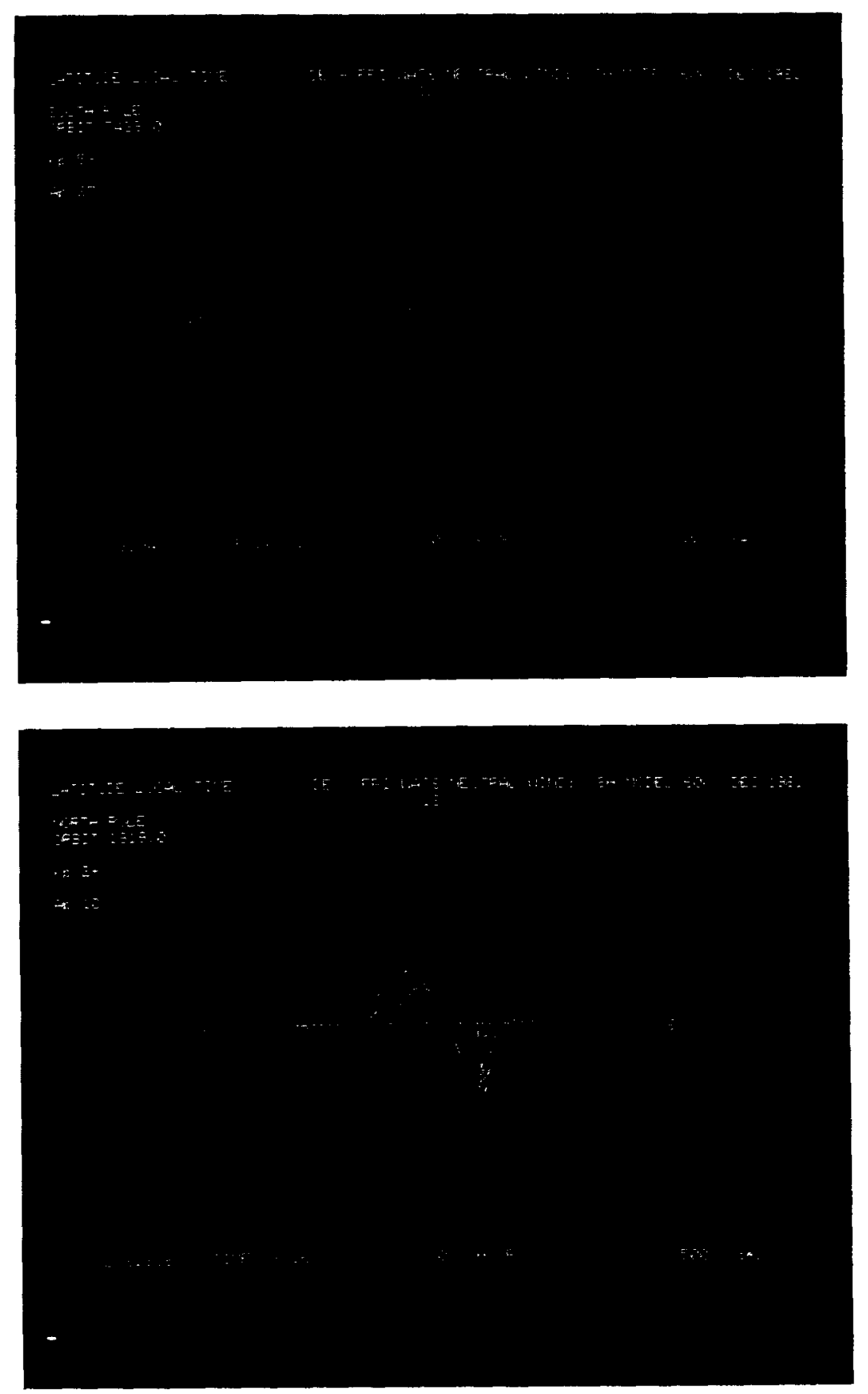

Fig. 2. Polar projections (solar local time and geographic latitude from pole to 40 degrees) illustrating the VSH model wind field (cyan arrows) for a) the Northern high-latitude region and b) the southern high-latitude region. The VSH wind vectors were calculated, in each case, at $400 \mathrm{~km}$ and for the UT appropriate to the DE 2 observations shown in yellow. The solar terminator is given by the curved blue line. Dotted yellow arrows indicate sections of the DE 2 pass for which there were no FPI (meridional) wind measurements available. The wind scale is shown at lower right.

To provide a more fully global perspective of the VSH model winds, we show in figure 3 a cartesian plot of global vector winds calculated using the VSH subroutine at the $400 \mathrm{~km}$ altitude level. Also shown for comparison are the DE 2 vector wind measurements made between altitudes of $-350-500 \mathrm{~km}$ during orbit number 7431 . The measured vector winds again illustrate reasonable qualitative agreement with the VSH model calculations in all regions, including the mid-latitude, high-latitude and equatorial regions. In general, for this orbit, the measured high-latitude winds were larger in magnitude than the VSH model values, although the reversal boundaries and the locations of the main morphological structures are in good agreement. Once again, our conclusion is that the VSH winds provide reasonable values (at least at upper thermospheric altitudes) that compare well with DE 2 global-scale measurements made during moderately-active geomagnetic conditions. 


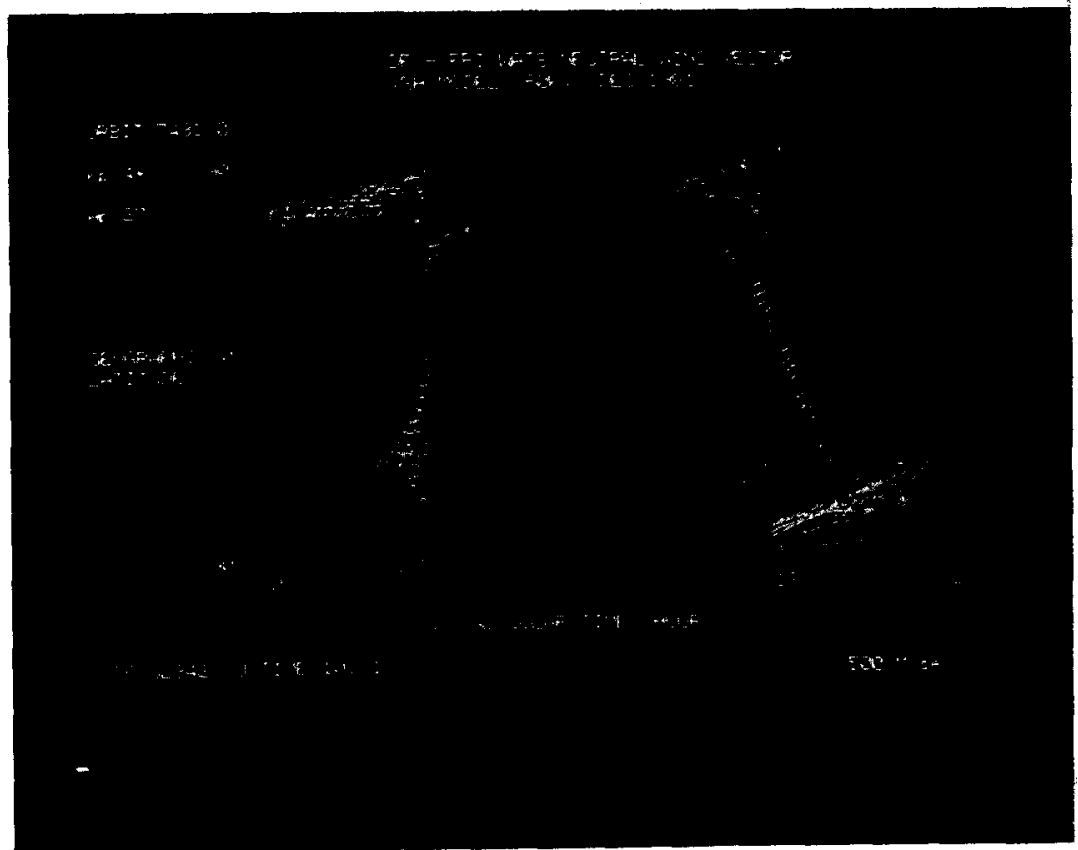

Fig. 3. Cartesian plot (geographic latitude and local solar time), illustrating the VSH global vector wind field at $400 \mathrm{~km}$ (cyan arrows). Also shown for comparison are the observed vector winds (in yellow) from orbit 7431 of DE 2 . The wind scale is to lower right and the curved red line represents the solar terminator.

A third example of VSH wind comparisons with DE 2 data is shown in figure 4 . Here, we plot averaged zonal wind measurements made using the WATS instrument during 1981 and 1982 for measurements taken within $5^{\circ}$ latitude of the geographic equator. These results are taken from the paper of Wharton et al. /8/ and depict the average of many orbits of data. The VSH wind results are shown by the solid line together with a low-order fourier fit to the data made by Herrero and Mayr /32/. It can be seen that the VSH zonal winds provide a good first-order fit to the averaged WATS data at all local times, with a small phase lead.

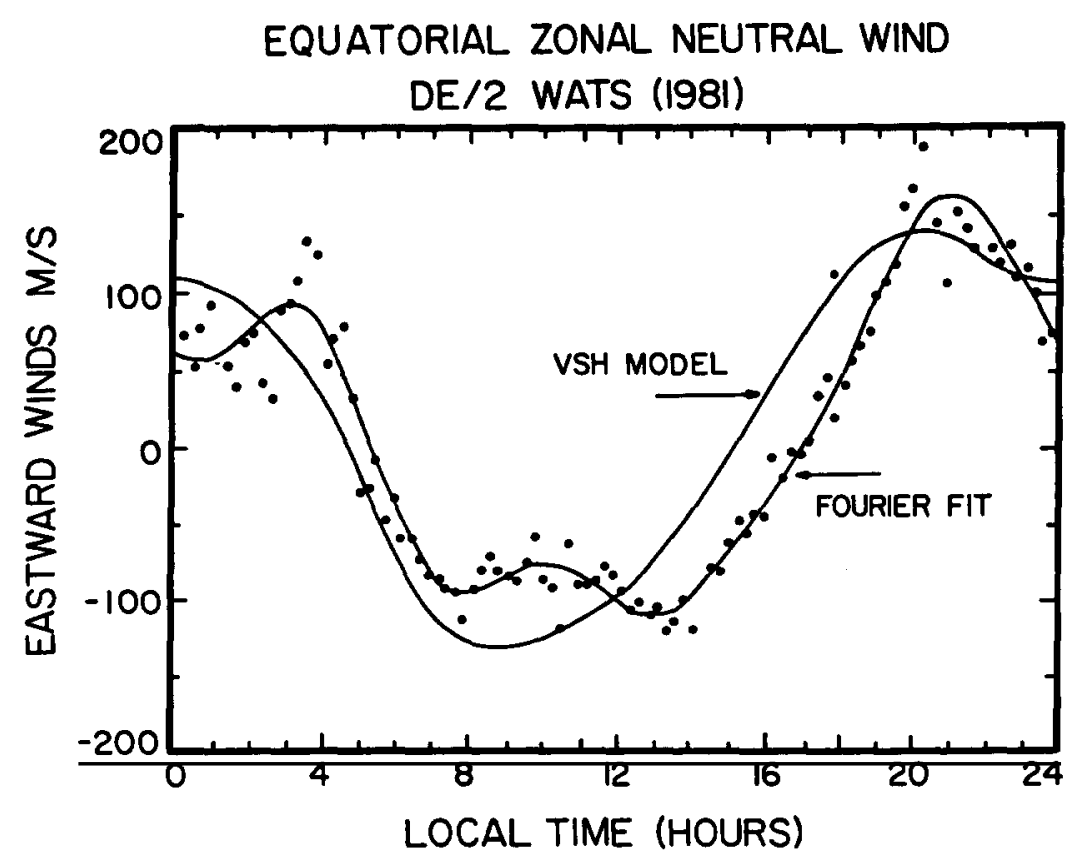

Fig. 4. Averaged equatorial zonal wind measurements from the WATS instrument on DE 2 taken from the paper by Wharton et al. $/ 33 /$. The results are plotted as a function of local solar time and correspond to data taken within $\pm 5^{\circ}$ latitude of the equator. 
As a further example of the utility of the VSH model, we show in Figures 5 and 6 the VSH model calculations for (a) zonal wind, (b) meridional wind and (c) temperature, calculated as a function of UT and altitude over locations at the geographic equator (Figure 5 ) and at Svalbard, Norway, $\left(78.2^{\circ} \mathrm{N}, 15.6^{\circ} \mathrm{W}\right.$ ) (Figure 6). Also shown for comparison in these figures are (d) the MSIS-83 temperatures for the same solar maximum, December solstice conditions. These figures serve to illustrate the altitude structure given by the computer model for a low-latitude location and a high-latitude location. It can be seen that the altitude variations of the VSH wind and temperature results have the required asymptotic form. There are, unfortunately, no global-scale measurements of lower thermosphere winds available with which to compare the results at altitudes less than $-250 \mathrm{~km}$ and the validity of the VSH model here is, therefore, linked solely to the confidence placed in the parent NCAR-TGCM calculations. Since the NCAR-TGCM solves the full set of "primitive equations" for thermospheric dynamics and since it has proved remarkably successful in the experimental tests at upper thermospheric altitudes, we believe that the VSH model provides valuable estimates for the entire altitude profile of thermospheric winds.

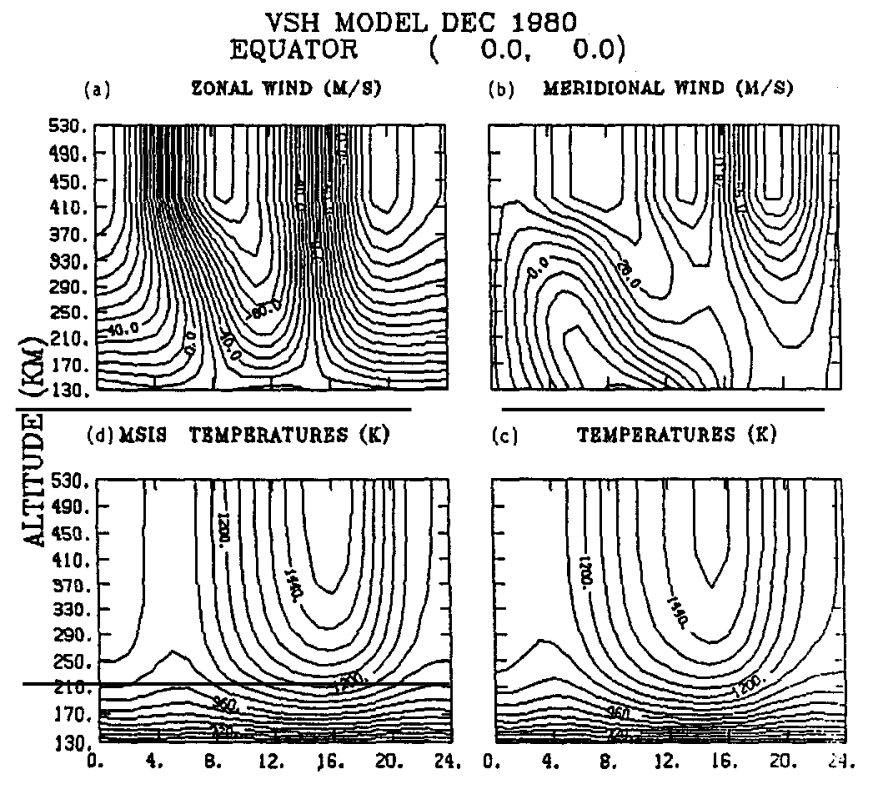

UNIVERSAL TIME (HOURS)

Fig. 5. Contour plots illustrating the altitudinal and UT-dependent variation of (a) the zonal wind, (b) the meridional wind, and (c) the temperature from the VSH model for calculations made at a location on the equator (latitude 0.0 , longitude 0.0 ). Also shown (d) are results from the MSIS-83 model for solar maximum December solstice conditions.

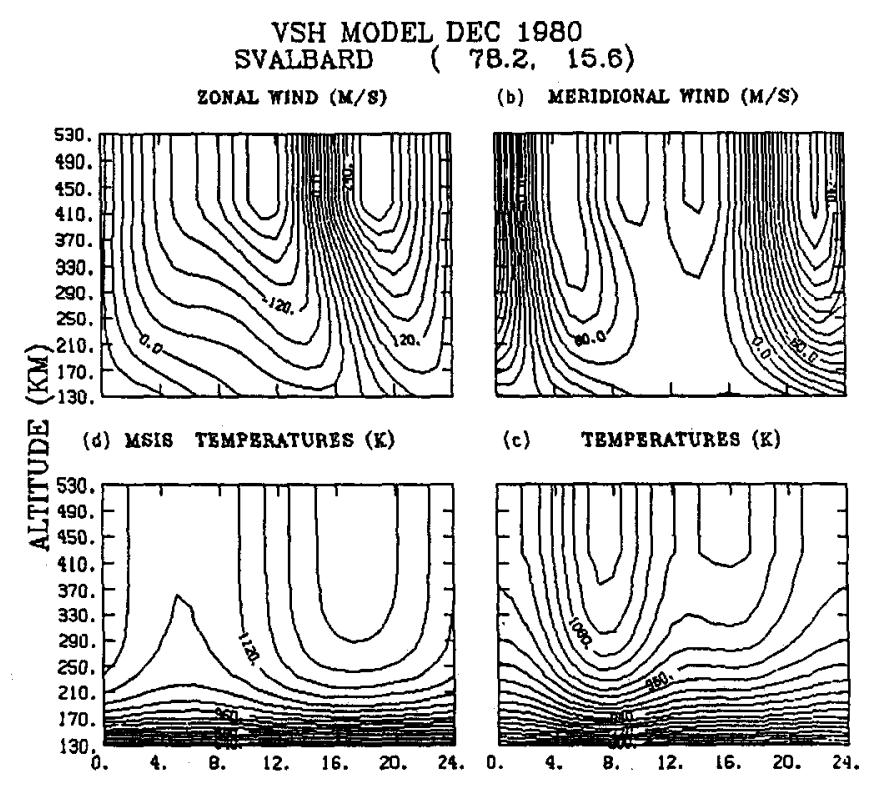

UNIVERSAL TIME (HOURS)

Fig. 6. Same as for Figure 5 except for the location at Svalbard, Norway (78.2 latitude, 15.6 longitude). 
The comparisons between MSIS- 83 and VSH model temperatures, shown in Figures $5 \mathrm{c}$ and $\mathrm{d}$ and $6 \mathrm{c}$ and $\mathrm{d}$, are of interest since they point out the major difference between the two model profiles. For the equatorial location shown in Figure 5, the MSIS-83 and VSH temperature profiles are in excellent agreement, as would be expected since MSIS-83 provides the background model input atmosphere for the initial "spin-up" of the NCAR-TGCM. There are, however, appreciable differences evident between the VSH temperature contours and the corresponding MSIS-83 profiles at the high-latitude Svalbard location, shown in Figure 6c and d. The reason for the apparent discrepancy here is related to two factors, (1) the different number of spectral harmonics used in the MSIS- 83 and VSH representations, and (2) the high-latitude heat source due to soft particle precipitation in the dayside cusp region employed in the NCAR-TGCM. MSIS-83 uses relatively few spherical harmonics and is, therefore, limited in the extent to which it can model local structures in the temperature field. The VSH model, on the other hand, uses $\sim 3$ times as many harmonics for the synthesis of its temperature field and is thereby able to model relatively smaller-scale features. The basic difference between the shapes of the MSIS-83 and VSH temperature contours in Figure 6 is due to the passage of the Svalbard location through the dayside cusp region near 0500-0900 hours UT. The NCAR-TGCM employs a high-altitude soft particle heat source in this region as discussed by Roble et al. $26 /$, leading to a significant local, high-altitude temperature enhancement. The VSH model reflects this modeled dayside cusp high altitude heating, while the MSIS-83 model does not show the feature.

We conclude that the VSH model, by virtue of the relatively large number of harmonics retained in order to model the high-latitude wind structure, is capable of modeling local temperature features that are filtered out from models that use fewer harmonics. It remains an open experimental question whether features such as the cusp temperature enhancement illustrated in Figure 6 are indeed present in spacecraft data sets.

\section{SUMMARY}

A computer model of the thermospheric wind and temperature fields, based on output from the NCAR-TGCM, has been constructed using a vector spherical harmonic expansion technique. The model is coded in a portable FORTRAN subroutine that reads in a set of coefficients containing the diurnal, altitudinal and spatial information necessary to synthesize the global wind and temperature fields. The current version of the model is valid for solar maximum, December solstice conditions. It has applicability for users who require reasonable values of neutral winds and temperatures in theoretical or comparative experimental studies. Comparisons with DE 2 observations have been used to illustrate the validity of the wind results at upper thermospheric altitudes.

The model framework can be extended to incorporate seasonal, solar cyclical and geomagnetic activity dependencies simply by running the NCAR-TGCM for specified geophysical conditions and calculating a set of spectral coefficient arrays. We plan to create a family of coefficient arrays in this manner that will provide reasonable wind and temperature results for a systematic range of conditions (solstice, equinox, solar minimum, active, moderate, quiet, disturbed, etc.) The retrieval subroutine will be common to these coefficient arrays. We also plan to use the VSH model framework to develop a semi-empirical model of thermospheric winds by appropriately merging observational data from DE 2 with the TGCM gridded calculations prior to performing the vector spherical harmonic expansion described in this paper. This work is underway.

\section{ACKNOWLEDGEMENTS}

We acknowledge support from NSF grant numbers ATM-8412828 and ATM-8610085 and NASA grant number NAG5-465 to the University of Michigan. This work was partially performed when one of the authors (TLK) was a visiting scientist at the High Altitude Observatory of the National Center for Atmospheric Research. Support from the Observatory is gratefully acknowledged. The authors are particularly grateful to Dr. P. Swartztrauber of NCAR for invaluable assistance with the vector spherical harmonic expansion and to Drs. H. G. Mayr, A. E. Hedin, R. E. Dickinson and A. D. Richmond for generously-given advice and consultations.

\section{REFERENCES}

1. Hays, P. B., T. L. Killeen, and B. C. Kennedy, The Fabry-Perot interferometer on Dynamics Explorer, Space Sci. Instrum., $5,395-416,1981$

2. Spencer, N. W., L. E. Wharton, H.B. Niemann, A. E. Hedin, G. R. Carignan, and J. C. Maurer, The Dynamics Explorer wind and temperature spectrometer, Space Sci. Instrum., 5, 417-428, 1981.

3. Killeen, T. L., P. B. Hays, N. W. Spencer, and L. E. Wharton, Neutral winds in the polar thermosphere as measured from Dynamics Explorer, Geophys. Res. Lett., 9, 957-960, 1982.

4. Killeen, T. L., P. B. Hays, G. R. Carignan, R. A. Heelis, W. B. Hanson, N. W. Spencer, and L. H. Brace, Ion-neutral coupling in the high-latitude F-region: Evaluation of ion heating terms from Dynamics Explorer 2, J. Geophys. Res., 89, 7495-7508, 1984a.

5. Killeen, T. L., R. W. Smith, P. B. Hays, N. W. Spencer, L. E. Wharton, and F. G. McCormac, Neutral winds in the high-latitude winter F-region: Coordinated observations from ground and space, Geophys. Res. Lett., 11, 311-314, 1984b.

6. Killeen, T. L., R. G. Roble, R. W. Smith, N. W. Spencer, J. W. Meriwether, D. Rees, G. Hernandez, P. B. Hays, L. L. Cogger, D. P. Sipler, M. A. Biondi, and C. A. Tepley, Mean neutral circulation in the winter polar F-region, J. Geophys. Res., 91, 1633-1649, 1986.

7. Spencer, N.W., L. E. Wharton, G. R. Carignan, and J. C. Maurer, Thermosphere zonal winds, vertical motions and temperature as measured from Dynamics Explorer, Geophys. Res. Lett. 9, 953-956, 1982.

8. Wharton, L. E., N. W. Spencer, and H. G. Mayr, The Earth's thermospheric superrotation from Dynamics Explorer 2, Geophys. Res. Lett. 11, 531-533, 1984

9. Hays, P. B., T. L. Killeen, N. W. Spencer, L. E. Wharton, R. G. Roble, B. E. Emery, T. J. Fuller-Rowell, D. Rees, L. A. Frank, and J. D. Craven, Observations of the dynamics of the polar thermosphere, J. Geophys. Res, 89, 5597, 1984.

10. Dickinson, R. E., E. C. Ridley, and R. G. Roble, A three-dimensional general circulation model of the thermosphere, J. Geophys. Res., 86, 1499-1512, 1981. 
11. Roble, R. G., R. E. Dickinson, and E. C. Ridley, Global circulation and temperature structure of the thermosphere with high-latitude plasma convection, J. Geophys. Res., 87, 1599-1614, 1982.

12. Fuller-Rowell, T. J., and D. Rees, A three dimensional time dependent global model of the thermosphere, J. Atmos. Sci., 37, $2545-2567,1980$.

13. Roble, R. G., R. E. Dickinson, E. C. Ridley, B. A. Emery, P. B. Hays, T. L. Killeen, and N. W. Spencer, The high latitude circulation and temperature structure of the thermosphere near solstice, Planet. Space Sci., 3l, 1299-1314, 1983

14. Roble, R. G., B. A. Emery, R. E. Dickinson, E. C. Ridley, T. L. Killeen, P. B. Hays, G. R. Carignan, and N. W. Spencer, Thermospheric circulation, temperature and compositional structure of the southern hemisphere polar cap during OctoberNovember, 1981, J. Geophys. Res., 89, 9057-9068, 1984.

15. Rees, D., T. J. Fuller-Rowell, R. Gordon, T. L. Killeen, P. B. Hays, L. E. Wharton, and N. W. Spencer, A comparison of wind observations of the upper thermosphere from the Dynamics Explorer satellite with the predictions of a global time-dependent model, Plant. Space Sci., 31, 1299-1314, 1983.

16. Rees, D., R. Gordon, T. J. Fuller-Rowell, M. Smith, G. R. Carignan, T. L. Killeen, P. B. Hays, and N. W. Spencer, The composition, structure, temperature and dynamics of the upper thermosphere in the polar regions during October to December, 1981, Planet. Space Sci., 33, 617-666, 1985.

17. Rees, D., T. J. Fuller-Rowell, R. Gordon, J. P. Heppner, N. C. Maynard, N. W. Spencer, L. E. Wharton, P. B. Hays, and T. L. Killeen, A theoretical and empirical study of the response of the high-latitude thermosphere to the sense of the "Y" component of the interplanetary magnetic field, Planet. Space Sci., in press, 1986.

18. Mayr, H. G., I. Harris, F. Varosi, and F. A. Herrero, Global excitation of wave phenomena in a dissipative, multi-constituent medium, 1, Transfer Function of the Earth's Thermosphere, J. Geophys. Res., 89, 10929, 1984.

19. Hedin, A. E., A revised thermospheric model based on mass spectrometer and incoherent scatter data: MSIS-83. J. Geophys Res., 88, 73-83, 10170-10188, 1983.

20. Killeen, T. L., and R. G. Roble, An analysis of the high latitude thermospheric wind pattern calculated by a thermospheric general circulation model, 1, Momentum forcing, J. Geophys. Res., 89, 7509-7522, 1984.

21. Dickinson, R. E., E. C. Ridley and R. G. Roble, Thermospheric general circulation with coupled dynamics and composition J. Atmos. Sci., 41 205-219, 1984

22. Hinteregger, H. E., Representations of solar EUV fluxes for aeronomical purposes, Adv. Space Res., 86, 801-813, 1981.

23. Torr, M. R., D. G. Torr, and H. E. Hinteregger, Solar flux variability in the Schumann-Runge continuum as a function of solar cycle 21, J. Geophys. Res., 85, 6063-6068, 1980.

24. Heelis, R. A., J. K. Lowell, and W. T. Kasprzak, A model of the high-latitude ionospheric convection pattern, J. Geophys. Res., 87, 6339-6345, 1982

25. Chiu, Y. T., An improved phenomenological model of ionospheric density, J. Atmos. Terr. Phys., 37, 1563-1570, 1975.

26. Roble, R. G., E. C. Ridley, and R. E. Dickinson, An auroral model for the NCAR Thermospheric General Circulation Model, J. Geophys. Res., submitted, 1986.

27. Spiro, R. W., P. H. Reiff, and L. J. Maher, Precipitating electron energy flux and auroral zone conductances - an empirical model, J. Geophys. Res., 87, 8215-8227, 1982.

28. Whalen, J. A., A quantitative description of the spatial distribution and dynamics of the energy flux in the continuous aurora, J. Geophys., Res., 88, 7155-7169, 1983.

29. Sica, R. J., M. H. Rees, G. J. Romick, G. Hernandez, and R. G. Roble, Auroral zone thermospheric dynamics: 1 Averages, J. Geophys. Res., 91, 3231-3244, 1986.

30. Bates, D. R., Some problems concerning the terrestrial atmosphere above about the $100 \mathrm{~km}$ level, Proc. R. Soc. London, Ser. $A, 253,451-462,1959$

31. Walker, J. C. G., Analytic representation of upper atmosphere densities based on Jacchia's static diffusion models, J. Atmos. Sci., 22, 462, 1965 .

32. Herrero, F. A. and H. G. Mayr, Tidal decomposition of zonal neutral and ion flows in the Earth's upper equatorial thermosphere, Geophys. Res. Lett., 13, 359, 1986. 